\title{
Theoretical and experimental characterization of emission and transmission spectra of Cerenkov radiation generated by ${ }^{177} \mathrm{Lu}$ in tissue
}

Nallely P. Jiménez-Mancilla

Keila Isaac-Olivé

Eugenio Torres-García

Miguel A. Camacho-López

Gerardo J. Ramírez-Nava

Héctor J. Mendoza-Nava 


\title{
Theoretical and experimental characterization of emission and transmission spectra of Cerenkov radiation generated by ${ }^{177} \mathrm{Lu}$ in tissue
}

\author{
Nallely P. Jiménez-Mancilla, ${ }^{\mathrm{a}, \star}$ Keila Isaac-Olivé, ${ }^{\mathrm{b}}$ Eugenio Torres-García, ${ }^{\mathrm{c}}$ Miguel A. Camacho-López, ${ }^{\mathrm{b}}$ \\ Gerardo J. Ramírez-Nava, ${ }^{d}$ and Héctor J. Mendoza-Navad \\ ${ }^{a}$ CONACyT, Instituto Nacional de Investigaciones Nucleares, Ocoyoacac, Estado de México, Mexico \\ bUniversidad Autónoma del Estado de México, Facultad de Medicina, Laboratorio de Fotomedicina, Biofotónica y Espectroscopía Láser \\ de Pulsos Ultracortos, Toluca, Estado de México, Mexico \\ 'Universidad Autónoma del Estado de México, Facultad de Medicina, Laboratorio de Simulación Monte Carlo y Dosimetría, Toluca, \\ Estado de México, Mexico \\ ${ }^{d}$ Instituto Nacional de Investigaciones Nucleares, Ocoyoacac, Estado de México, Mexico
}

\begin{abstract}
Cerenkov radiation (CR) is the emission of UV-vis light generated by the de-excitation of the molecules in the medium, after being polarized by an excited particle traveling faster than the speed of light. When $\beta$ particles travel through tissue with energies greater than $219 \mathrm{keV}, \mathrm{CR}$ occurs. Tissues possess a spectral optical window of 600 to $1100 \mathrm{~nm}$. The CR within this range can be useful for quantitative preclinical studies using optical imaging and for the in-vivo evaluation of ${ }^{177} \mathrm{Lu}$-radiopharmaceuticals ( $\beta$-particle emitters). The objective of our research was to determine the experimental emission light spectrum of ${ }^{177} \mathrm{Lu}-\mathrm{CR}$ and evaluate its transmission properties in tissue as well as the feasibility to applying CR imaging in the preclinical studies of ${ }^{177} \mathrm{Lu}$-radiopharmaceuticals. The theoretical and experimental characterizations of the emission and transmission spectra of ${ }^{177} \mathrm{Lu}-\mathrm{CR}$ in tissue, in the vis-NIR region (350 to $900 \mathrm{~nm}$ ), were performed using Monte Carlo simulation and UV-vis spectroscopy. Mice ${ }^{177}$ Lu-CR images were acquired using a charge-coupled detector camera and were quantitatively analyzed. The results demonstrated good agreement between the theoretical and the experimental ${ }^{177} \mathrm{Lu}-\mathrm{CR}$ emission spectra. Preclinical CR imaging demonstrated that the biokinetics of ${ }^{177}$ Lu-radiopharmaceuticals in the main organs of mice can be acquired. $\odot$ The Authors. Published by SPIE under a Creative Commons Attribution 4.0 Unported License. Distribution or reproduction of this work in whole or in part requires full attribution of the original publication, including its DOI. [DOI: 10.1117/1.JBO.24.7.076002]
\end{abstract}

Keywords: Cerenkov radiation; ${ }^{177}$ Lu-radiopharmaceuticals; emission and light transmission in tissue; optical imaging; preclinical studies.

Paper 190081RR received Mar. 27, 2019; accepted for publication Jun. 20, 2019; published online Jul. 16, 2019.

\section{Introduction}

Cerenkov radiation (CR) is the energy produced in the ultraviolet (UV)-visible region of the electromagnetic spectrum, when the charged particles in a medium travel faster than the speed of light in the same medium. Because of the high particle velocity, the molecules of the medium are polarized and are immediately de-excited by releasing energy in the form of UV and visible radiation. CR generation is related to the velocity and energy of the particles as well as the refractive index of the environment. The threshold condition for CR generation is $v_{p}>c / n$, where $n$ is the refractive index of the medium, and $v_{p}$ and $c$ are the speed of the particle and the speed of light in vacuum, respectively. ${ }^{1,2}$ For $\beta$ particles traveling in water $(n=1.33)$, the energy threshold for CR generation is $261 \mathrm{keV}$, and it changes in muscle tissue $(n=1.4)$ to $219 \mathrm{keV}^{3}$

The variability of the tissue refractive index plays an important role in the production of photons through CR. The shape of the CR spectrum is independent of the radionuclide, but the intensity is regulated by the $\beta$ particle source. Therefore, the $\mathrm{CR}$ intensity is determined by the radionuclide radioactive decay

*Address all correspondence to Nallely P. Jiménez-Mancilla, E-mail: nallely_ jimenez@yahoo.com.mx scheme and the number of transitions per unit time (activity). For example, $\beta$ particles with higher end-point energy $\left(E_{\max }\right)$ will result in the emission of more Cerenkov photons. ${ }^{4}$

The optical imaging of radiopharmaceuticals using CR is a preclinical molecular imaging modality that describes the distribution, in time and space, of the charged-particle emitters through the tissue, using highly sensitive charge-coupled detectors (CCDs). ${ }^{5,6}$ This imaging modality has advantages, such as lower infrastructure cost, higher resolution, and lower image acquisition time, compared to micropositron-emission tomography (PET), ${ }^{7,8}$ a conventional nuclear imaging technique widely used.

${ }^{177} \mathrm{Lu}$ is a radionuclide $\beta$ emitter, with a half-life of 6.71 days and $E_{\max }$ emission of $0.497 \mathrm{MeV}(78 \%)$. It has been successfully used for radiopeptide therapy with an efficient crossfire effect in cancer cells. ${ }^{9,10}$ Hence the development of new ${ }^{177} \mathrm{Lu}$-radiopharmaceuticals is of immense interest in the global medical field. ${ }^{11}$

Some of the $\beta$ particles emitted by ${ }^{177} \mathrm{Lu}$ reach the relativistic limit because they travel faster than the speed of light in different media. Nevertheless, to successfully use ${ }^{177} \mathrm{Lu}$ for applications in preclinical CR imaging, it is necessary to establish several physical parameters, such as the ${ }^{177} \mathrm{Lu}-\mathrm{CR}$ emission spectrum in the tissue optical window (600 to $1100 \mathrm{~nm}),{ }^{12}$ number of photons produced per unit of ${ }^{177} \mathrm{Lu}$ activity considering the loss of 
energy in its path through the tissue, ${ }^{177} \mathrm{Lu}-\mathrm{CR}$ transmission spectrum in the tissue, and the maximum depth at which the ${ }^{177} \mathrm{Lu}$ source should be located in the tissue to obtain CR signals suitable for imaging. These parameters, so far unknown, determine the applicability of ${ }^{177} \mathrm{Lu}-\mathrm{CR}$ in the preclinical assessment (biokinetic models) of ${ }^{177}$ Lu-radiopharmaceuticals, based on the optical images obtained in murine models. .,2,13,14 $^{2}$

The objective of this work was to determine the experimental emission light spectrum of ${ }^{177} \mathrm{Lu}-\mathrm{CR}$, and evaluate its transmission properties in tissue as well as the feasibility of using CR imaging in the preclinical studies of ${ }^{177} \mathrm{Lu}$-radiopharmaceuticals. For this purpose, the theoretical and experimental characterizations of the emission and transmission of CR generated by ${ }^{177} \mathrm{Lu}$ in tissue were performed using the Frank-Tamm and relativistic theory, Monte Carlo simulation (MCLTmx code), and UV-vis spectroscopy techniques. The ${ }^{177} \mathrm{Lu}-\mathrm{CR}$ images of mice were acquired using a CCD camera. The total number of photons per unit time per unit area $\left(\mathrm{p} / \mathrm{s} / \mathrm{mm}^{2}\right)$ was quantitatively analyzed in each region of interest to obtain the total number of disintegrations, $N\left(\left[\mathrm{MBq}^{*} \mathrm{~h} / \mathrm{MBq}\right]\right)$, and the biokinetic models in the kidneys and tumor regions.

\section{Materials and Methods}

\subsection{Theoretical Characterization of ${ }^{177} \mathrm{Lu}-\mathrm{CR}$}

From the emission spectrum of ${ }^{177} \mathrm{Lu} \beta$ particles $\left(E_{\max }=\right.$ $0.497 \mathrm{MeV}$ ), the threshold energies required for $\mathrm{CR}$ generation in water and tissue were determined using Eq. (1): ${ }^{4}$

$v_{p}>\frac{c}{n}$,

where $c$ is the speed of light in vacuum, $n$ is the refractive index of the medium, and $v_{\mathrm{p}}$ is the speed of the emitted $\beta$ particle, which can be calculated using Eq. (2). Here, $E_{0}$ is the energy of the $\beta$ particle at rest and $E$ is the energy of the $\beta$ particle:

$v_{p}=c\left[1-\frac{E_{0}^{2}}{\left(E+E_{0}\right)^{2}}\right]^{1 / 2}$.

The number of photons produced $(\partial N)$ over the distance traveled $(\partial x)$ by the $\beta$ particle was obtained using the Frank-Tamm formula depicted in Eq. (3), ${ }^{15}$ which mathematically describes the light dependence with respect to the radionuclide energy and refractive index of the medium

$$
\frac{\partial N}{\partial x}=2 \pi \alpha\left(1-\frac{1}{B^{2} n^{2}}\right) \int_{\lambda_{1}}^{\lambda_{2}} \frac{1}{\lambda^{2}} \mathrm{~d} \lambda,
$$

where $\alpha$ is the fine-structure dimensionless constant (1/137), $B$ is the ratio of the particle velocity to the speed of light, $n$ is the refractive index of the medium, and $\lambda_{1}$ and $\lambda_{2}$ are the inferior and superior wavelengths of the emission spectrum, respectively.

The mean range $(R)$ of the charged particles is an extensively used parameter in nuclear medicine. ${ }^{16}$ Here, $R$ is defined as the average linear scope of the charged particle in a material medium; for $\beta$ particles, it can be determined using Eqs. (4) and (5):

$$
R_{m}=412 E_{\max }^{1.265-0.0954 \ln E_{\max }} 0.01<E_{\max }<2.5 \mathrm{MeV},
$$

$R_{m}=530 E_{\max }-106 E_{\max }>2.5 \mathrm{MeV}$,

where $E_{\max }$ is the maximum energy of the emitted particle and $R_{m}$ is the mass range in milligram per square centimeter. To obtain the mean range in a medium or material, $R_{m}$ is divided by the density of the material $\left(\rho_{\text {water }}=1.00 \mathrm{~g} / \mathrm{cm}^{3}\right.$ and $\rho_{\text {tissue }}=1.07 \mathrm{~g} / \mathrm{cm}^{3}$ ).

\subsection{Experimental ${ }^{177}$ Lu-CR Emission Spectrum}

${ }^{177} \mathrm{Lu}$ was procured from ITG Isotope Technologies, with a radionuclidic purity of $99.9 \%$ and activity of $37 \mathrm{GBq}$ in $2 \mathrm{~mL}$ of 0.04 of hydrochloric acid $(\mathrm{HCl})$ within a glass ampoule vial. To characterize the CR emission spectrum, a BLUE-wave optical fiber spectrometer (StellarNet Inc.) was used; the spectrum was obtained within the 350 - to $900-\mathrm{nm}$ range.

An experimental setup was designed, including the construction of a lead-shielded insulated dark chamber with a circular aperture for the optical fiber. A glass ampoule vial was placed within the chamber and an optical fiber was placed in contact with the vial. This arrangement isolated the system from the background light and protected the involved personnel from the ${ }^{177} \mathrm{Lu}$ ionizing radiation.

The ${ }^{177} \mathrm{Lu}-\mathrm{CR}(\lambda)$ emission spectrum [gross spectrum, $\left.{ }^{177} \mathrm{Lu}-\mathrm{CR}_{\text {gross }}(\lambda)\right]$ was registered continuously in the 350 - to $900-\mathrm{nm}$ range. In addition, the background spectrum was monitored similarly. The transmission spectrum $(T)$ of the empty glass ampoule vial was also recorded in a Perkin Elmer spectrophotometer (model 551S) in the same wavelength range (350 to $900 \mathrm{~nm}$ ). The absorption spectrum of only one wall of the vial $\left(\mathrm{Abs}_{\mathrm{wall}}\right.$ vial $)$ was obtained as follows:

$\operatorname{Abs}_{\text {wall vial }}(\lambda)=-\log [\sqrt{T(\lambda)}]$

Finally, the absorption of the optical fiber $\left(\mathrm{Abs}_{\text {fiber }}\right)$ was also determined. In this case, the StellarNet spectrophotometer was illuminated with an aligned white light source and the transmission spectra in the presence $(I)$ and the absence $\left(I_{0}\right)$ of the optical fiber were collected. From these spectra, the absorption spectrum in the range from 350 to $900 \mathrm{~nm}$ was determined as follows:

$\operatorname{Abs}_{\text {fiber }}(\lambda)=-\log \frac{I(\lambda)}{I_{0}(\lambda)}$.

All obtained spectra were normalized. The final ${ }^{177} \mathrm{Lu}-\mathrm{CR}$ emission spectrum $\left[{ }^{177} \mathrm{Lu}-\mathrm{CR}(\lambda)\right]$ corrected by background, glass vial, and optical fiber absorption was obtained as follows:

$$
{ }^{177} \operatorname{Lu}-\mathrm{CR}(\lambda)=\frac{{ }^{177} \operatorname{Lu}-\mathrm{CR}_{\text {gross }}(\lambda)-\operatorname{Background}(\lambda)}{\operatorname{Abs}_{\text {wall vial }}(\lambda) * \operatorname{Abs}_{\text {fiber }}(\lambda)} .
$$

\subsubsection{Validation of the experimental ${ }^{177}$ Lu-CR emission spectrum}

The experimental ${ }^{177} \mathrm{Lu}-\mathrm{CR}(\lambda)$ emission spectrum was validated using the CR spectrum of ${ }^{68} \mathrm{Ga}$ reported by Thorek et al., ${ }^{2}$ because CR generation phenomenon does not depend on the type of radionuclide. Gallium-68 is a positron emitter that has a halflife of 68 min with a $\beta^{+}$particle $\left(E_{\max }=1.9 \mathrm{MeV}\right)$ abundance of $89 \%$. The validation was performed by a point-by-point 
comparison of the normalized CR values reported by Thorek et al. ${ }^{2}$ and the normalized values obtained from the experimental ${ }^{177} \mathrm{Lu}-\mathrm{CR}(\lambda)$ spectrum in the spectral range of 380 to $600 \mathrm{~nm}$. It is to be noted that Thorek et al. only reported the CR spectrum in the 300- to $600-\mathrm{nm}$ range; hence the ${ }^{177} \mathrm{Lu}-\mathrm{CR}(\lambda)$ emission spectrum emitted in the tissue optical window is unknown.

\subsection{Determination of the Optical ${ }^{177} \mathrm{Lu}-\mathrm{CR}$ Transmission in Tissue}

Initially, the light transmission profile through tissue was obtained by visible photon transport simulation using the MCLTmx code. ${ }^{17}$ The ${ }^{177}$ Lu-CR light transmission in tissue (transmission efficiency) was then obtained by the product of the experimental ${ }^{177} \mathrm{Lu}-\mathrm{CR}(\lambda)$ emission spectrum and the light transmission profile simulated by the Monte Carlo MCLTmx code at different wavelengths and tissue thicknesses. Finally, the percentage of total emergent photons from the tissue optical window was obtained as a sum of the integrals of each function of the ${ }^{177} \mathrm{Lu}-\mathrm{CR}(\lambda)$ transmission efficiency in the 600 - to 900-nm range.

\subsubsection{Light-transmission profile in tissue using the Monte Carlo MCLTmx code}

The Monte Carlo MCLTmx code simulates a monoenergetic and isotropic point source, because the beta emitter $\left({ }^{177} \mathrm{Lu}\right)$ was considered homogenously distributed within the interest region (tumor and kidneys). Since the beta emission is isotropic, $\mathrm{CR}$ emission is also isotropic.

The source was located at the center of a biological tissue plate of finite thickness and sides with the following girth at each side of the source: $0.05,0.1,0.2,0.3,0.4,0.5,0.6,0.7$, $0.8,0.9,1,1.2,1.4,1.5$, and $2 \mathrm{~cm}$. For each girth, photons with the following wavelengths were, respectively, generated: 350 , $400,450,500,550,600,650,700,750,800,850$, and $900 \mathrm{~nm}$.

The reduced dispersion coefficient $\left[\mu_{s}^{\prime}(\lambda)\right]$ was obtained using Eq. (9), and the anisotropy $[g(\lambda)]$ was calculated using ${ }^{18}$

$\mu_{s}^{\prime}(\lambda)=46\left[\frac{\lambda}{500 \mathrm{~nm}}\right]^{-1.421}$

$g(\lambda)=0.943-5.6 e^{-0.0084 \lambda}$.

Using Eqs. (9) and (10), the dispersion coefficient was obtained through Eq. (11). The absorption coefficient, as a function of the wavelength $\left[\mu_{a}(\lambda)\right]$, was obtained from the Svaasand1995 data reported by Lister et al., ${ }^{19}$ which can be considered an average of all biological tissue containing blood, and Eqs. (12) and (13):

$$
\begin{aligned}
& \mu_{s}(\lambda)=\frac{\mu_{s}^{\prime}}{1-g(\lambda)}, \\
& \mu_{a}(\lambda)=5.54+2939.68\left[\frac{32.7}{4(\lambda-414.8)^{2}+32.7^{2}}\right] \\
& \quad \text { if } 350 \leq \lambda<510(\mathrm{~nm}),
\end{aligned}
$$$$
2.30+1073.01\left[\frac{81.2}{4(\lambda-550)^{2}+81.2^{2}}\right] \text { if } 510 \leq \lambda \leq 900(\mathrm{~nm}) .
$$

The Svaasand-1995 data were reported for $\mu_{a}(\lambda)$, ranging from 350 to $750 \mathrm{~nm}$, which was extrapolated to obtain the $\mu_{a}(\lambda)$ values for wavelengths between 750 and $900 \mathrm{~nm}$ using Eq. (13).

Finally, the primary and secondary transmission data were collected through a tissue plate. A total of $10^{7}$ histories were run; three runs were conducted for each wavelength.

\section{$2.4{ }^{177}$ Lu-CR Image Acquisition and Quantitative Analysis}

T47D human breast cancer tumors were induced subcutaneously in 8-week-old athymic mice weighing $22 \mathrm{~g}$. All the studies on athymic mice were conducted according to the official Mexican norm, 062-ZOO-1999.

${ }^{177} \mathrm{Lu}$-radiopharmaceutical with a radiochemical purity of $>98 \%$ was administered. The preparation of the radiopharmaceutical was previously reported by Aranda-Lara. ${ }^{20}$

${ }^{177} \mathrm{Lu}-\mathrm{CR}$ and $\mathrm{x}$-ray images were acquired using Bruker's in-vivo xtreme (BVX) system which is a preclinical multimodal imaging system that records images using different modalities (radiographic, radioisotopic, fluorescence, and Cerenkov luminescence) through an ultrathin phosphor screen and CCD camera. All the images were acquired and reconstructed following the methodology reported by Ramírez-Nava. ${ }^{21}$ Briefly, CR and $\mathrm{X}$-ray images of the mice were acquired, as represented in Fig. 1, with BVX and its multimodal animal rotation system (MARS) after the administration of ${ }^{177} \mathrm{Lu}(2,4,24,48$, and $120 \mathrm{~h})$. Each ${ }^{177} \mathrm{Lu}-\mathrm{CR}$ acquisition gathered a set of 13 projections, covering the $360 \mathrm{deg}$ of rotation (30-deg steps). The acquisition time of each projection was $5 \mathrm{~min}$, the binning was $4 \times 4$, in a field of view (FOV) of $12.5 \mathrm{~cm}$.

The x-ray images were used to obtain the anatomical tomographic reconstruction of the rodents. These images were registered with a tube voltage of $45 \mathrm{kVp}$, a current of $497 \mu \mathrm{A}$, and an aluminum filter of $0.8 \mathrm{~mm}$. In this modality, a batch of

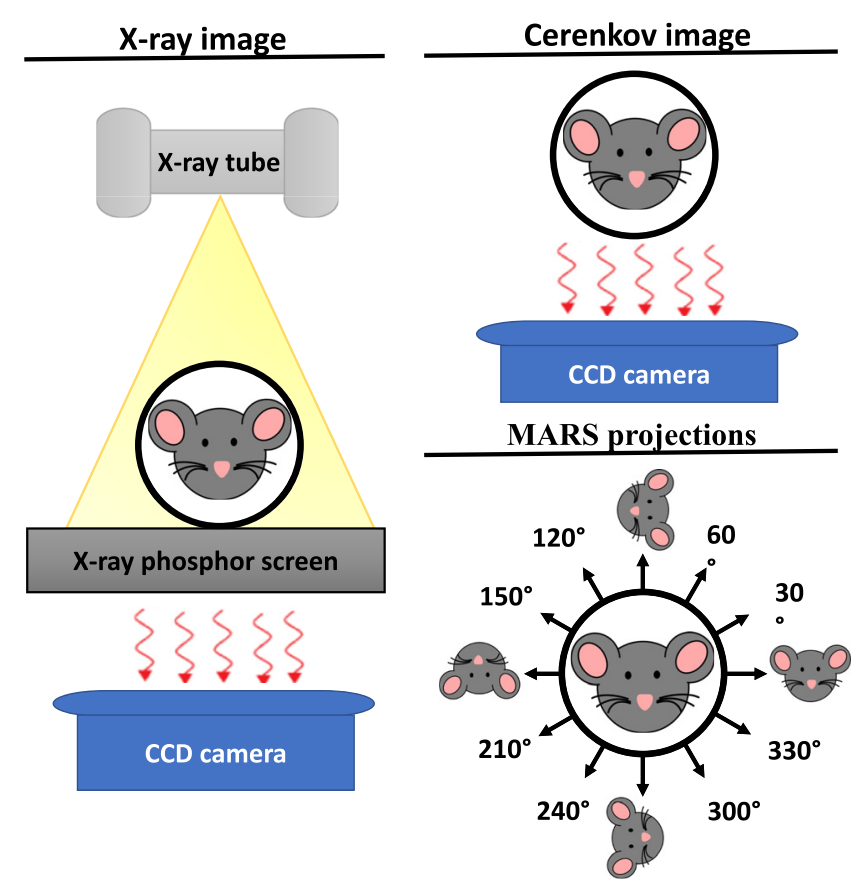

Fig. 1 Schematic representation of $\mathrm{x}$-ray and Cerenkov images acquisition using BVX system and projection images acquired with MARS. 
181 projections (360-deg coverage and 2-deg steps) was acquired. The acquisition time of each projection was $10 \mathrm{~s}$, using a $1 \times 1$ binning, and a $12.5-\mathrm{cm}$ FOV. Both CR and x-ray images were reconstructed with the filtered backprojection algorithm, which was implemented in MATLAB R2108b (MathWorks, 2018).

The regions of interest (kidneys and the tumor) were located in the planar images of mice. From the 13 projection images acquired with the MARS, the mean depth of the tumor and kidneys were determined. The ${ }^{177} \mathrm{Lu}-\mathrm{CR}$ images were quantitatively analyzed by counting the photons per unit time per unit area $\left(\mathrm{p} / \mathrm{s} / \mathrm{mm}^{2}\right)$ in each region of interest.

\subsubsection{Determination of the total number of $\mathrm{p} / \mathrm{s} / \mathrm{mm}^{2}$ as a function of the activity}

The total activity in the regions of interest was determined by calibrating the CCD camera, by counting the total number of
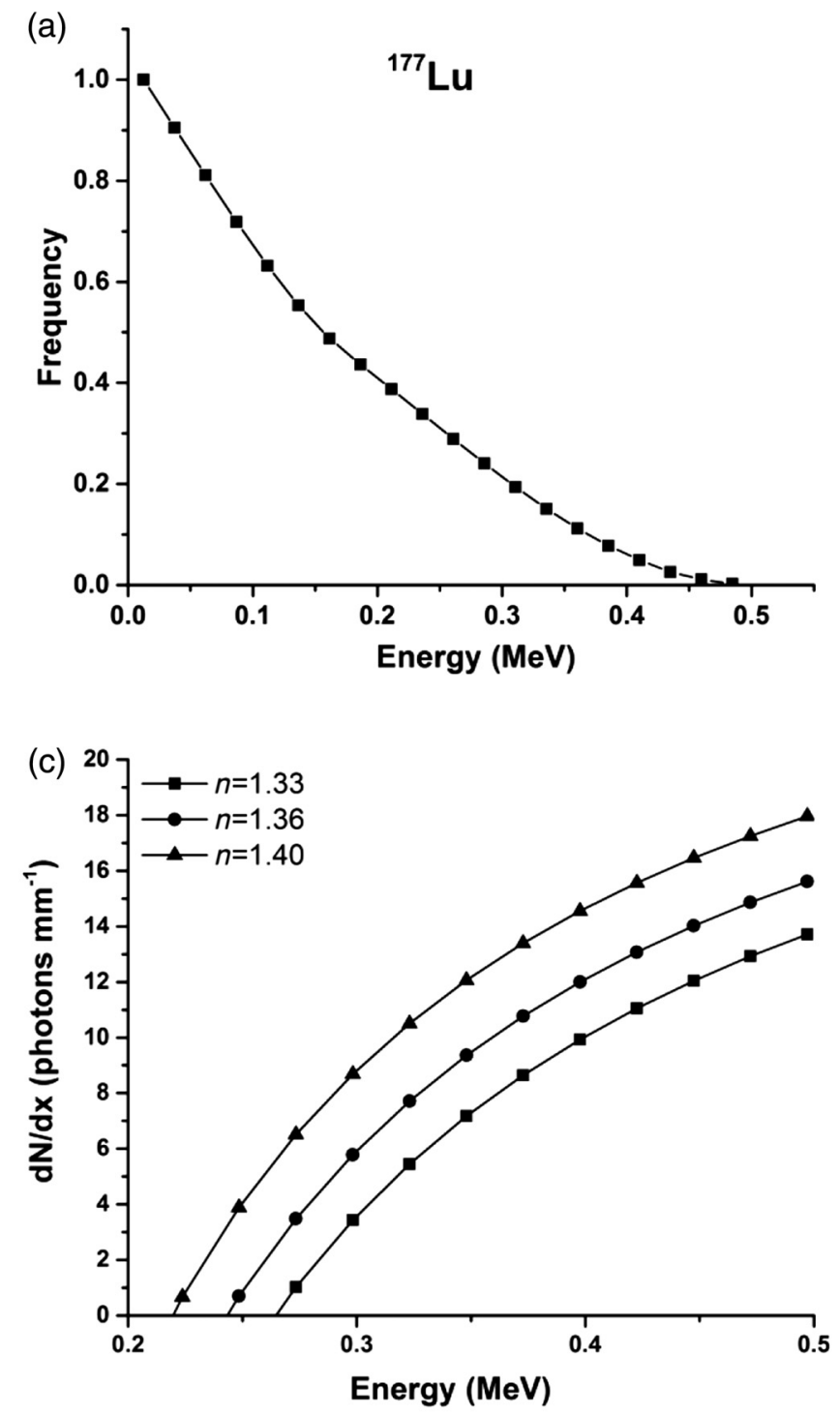

$\mathrm{p} / \mathrm{s} / \mathrm{mm}^{2}$ detected by it from different known radiation activities $(0.5,1,2,4,8$, and $16 \mathrm{MBq})$. The mathematical function describing $\mathrm{p} / \mathrm{s} / \mathrm{mm}^{2}$ as a function of the activity was then obtained.

Biokinetic models $\left[A_{h}(t)\right]$ for the tumor and kidneys were constructed using the activity registered at 2, 4, 24, 48, and $120 \mathrm{~h}$ postinjection in the regions of interest, and the number of total disintegrations $(N)$ normalized to the unit-administered activity (MBq) was obtained as follows:

$N_{\text {region }}=\int_{t=0}^{t=\infty} A_{h}(t) \mathrm{d} t$

The total number of disintegrations, $N_{\text {region }}$, obtained was compared with that obtained through a method involving the counting of the activity in a well-type scintillation detector from ex-vivo organs, reported by Aranda-Lara et al. ${ }^{20}$

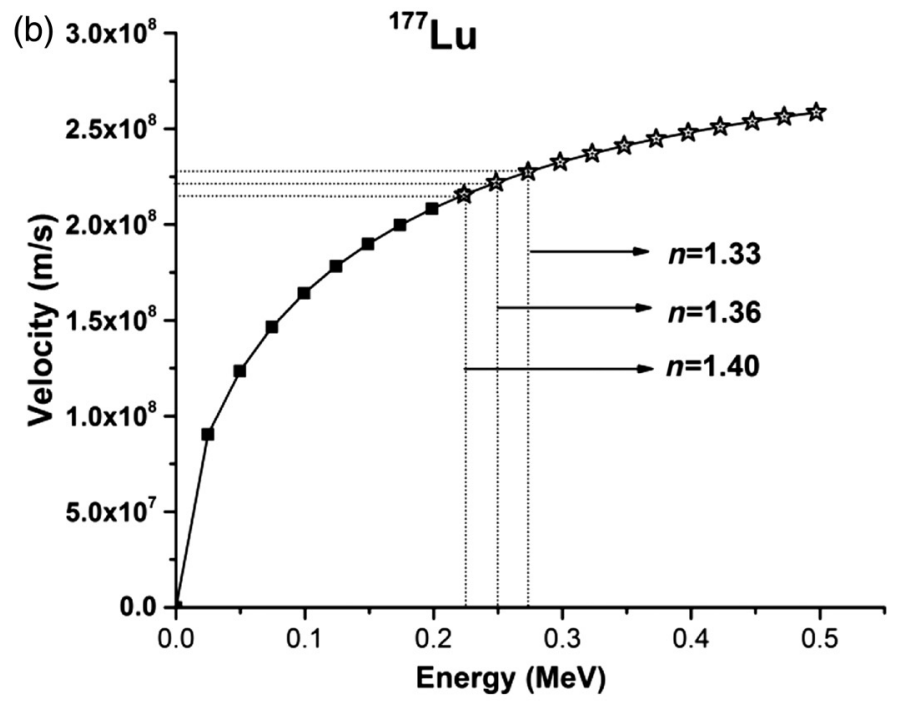

(d)

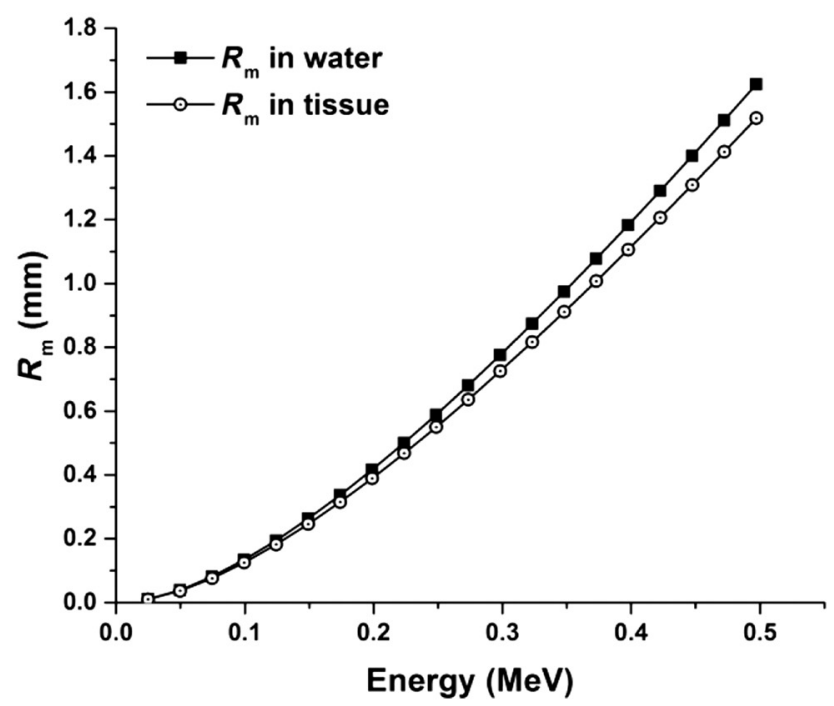

Fig. 2 (a) Energy spectrum of the $\beta$ particles emitted by ${ }^{177} \mathrm{Lu}$, reported by radiation dose assessment resource ${ }^{24}$ and (b) relationship between $\beta$ energy and their velocity in water $(n=1.33)$ or tissue $(n=1.34-1.36)$; the star represents the energy at which CR can be produced. (c) Number of photons produced by unit of $\beta$ particle traveled path obtained with the Frank-Tamm theory in water and tissue, and (d) mean ranges of $\beta$ particles emitted by ${ }^{177} \mathrm{Lu}$ in water and tissue determined by nuclear medicine technique. ${ }^{16}$ 


\section{Results and Discussion}

\subsection{Theoretical Characterization of ${ }^{177} \mathrm{Lu}-\mathrm{CR}$}

Figure 2 shows the theoretical characterization of the physical properties of ${ }^{177} \mathrm{Lu}$. Figure 2(a) presents the energy spectrum of the $\beta$ particles emitted by ${ }^{177} \mathrm{Lu}$; Fig. 2(b) depicts the relationship between the energy and velocity in water $(n=1.33)$ and the tissue ( $n=1.34$ to 1.36 ); Fig. 2(c) displays the number of photons produced by the traveling of the emitted $\beta$ particles for unit distance. This number was obtained using the Frank-Tamm equation in different media (water and tissue); Fig. 2(d) depicts the linear range $(R)$ of the $\beta$ particles in water and tissue. It can be observed that the threshold energy necessary for CR generation in water and tissue are $246.06 \mathrm{keV}$ and 219.19 to $242.97 \mathrm{keV}$, respectively, depending on the tissue refractive index. Around $25 \%$ of the $\beta$ particles emitted by ${ }^{177} \mathrm{Lu}$ presented an energy greater than the threshold energy required for CR generation. The $R$, where the $\beta$ particles can produce $\mathrm{CR}$, was $\sim 1 \mathrm{~cm}$ in water and $0.9 \mathrm{~cm}$ in tissue. This characteristic is important in image reconstruction because it enables the improvement of the spatial and temporal resolutions of the image by differentiating between the material interfaces. ${ }^{22,23}$ Moreover, the number of the total photons produced per unit of activity can be calculated by the functions shown in Fig. 2(c), corrected by the probability of emission of the $\beta$ particle [Fig. 2(a)]; in the case of water, the total numbers of photons by a unit of activity was 556,089 .

\subsection{Experimental ${ }^{177}$ Lu-CR Emission Spectrum}

Thorek et al. ${ }^{2}$ previously reported the Cerenkov spectrum of ${ }^{68} \mathrm{Ga}$, and since this spectrum does not depend on the type of radionuclide used, it is expected that the normalized $\mathrm{CR}$ spectrum of ${ }^{177} \mathrm{Lu}$ is equal to that of ${ }^{68} \mathrm{Ga}$. In a previous work, CR spectrum was recorded from ${ }^{68} \mathrm{Ga}$ but for wavelengths lower than $600 \mathrm{~nm}$. In this work, the area of interest is the optical window of the tissue $(\lambda>600 \mathrm{~nm})$, therefore CR spectrum was recorded from ${ }^{177} \mathrm{Lu}$ reaching the wavelength of $900 \mathrm{~nm}$.

Figure 3 shows the experimental CR emission spectrum, corrected for the background, glass vial, and optical fiber absorption. The spectral range of the tissue optical window

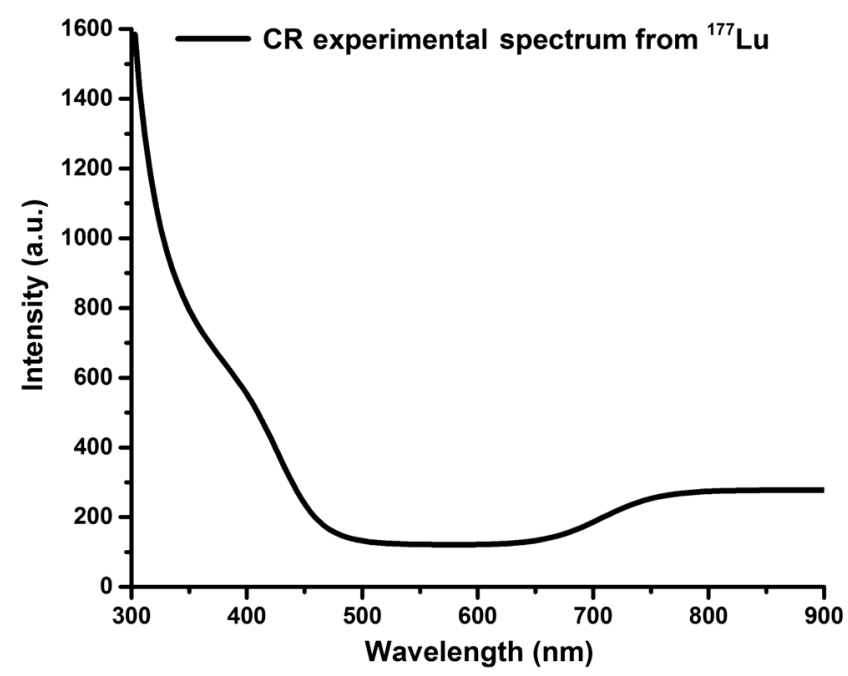

Fig. 3 Experimental CR spectrum, corrected for background, glass vial, and optical fiber absorption, obtained in the wavelength range from 300 to $900 \mathrm{~nm}$.
(600 to $900 \mathrm{~nm}$ ) is an important contributor to the total intensity of the spectrum.

Figure 4(a) shows the normalized emission spectrum of ${ }^{68} \mathrm{Ga}$ reported by Thorek et al., ${ }^{2}$ while Fig. 4(b) presents the normalized experimental ${ }^{177} \mathrm{Lu}-\mathrm{CR}$ spectrum of ${ }^{177} \mathrm{Lu}$ obtained in this study. Figure 4(c) depicts the comparison between the experimental ${ }^{177} \mathrm{Lu}-\mathrm{CR}$ spectrum and that reported by Thorek et al. ${ }^{2}$ The comparison does not show significant differences in the 380 to $600 \mathrm{~nm}$ spectral window $(<2 \%)$ because CR generation does not depend upon the radionuclide and only depends on the emission energy. Thus, this result validates the complete ${ }^{177} \mathrm{Lu}-\mathrm{CR}$ spectrum (380 to $900 \mathrm{~nm}$ ). The results of the normalized experimental characterization demonstrated that $45 \%$ of the total intensity of the ${ }^{177} \mathrm{Lu}-\mathrm{CR}$ emission spectrum corresponds to the photons within the tissue optical window, supporting possible applications for the acquisition of optical images similar to those of positron emitters (e.g., ${ }^{18} \mathrm{~F},{ }^{64} \mathrm{Cu}$, and ${ }^{89} \mathrm{Zr}$ ). ${ }^{8,25,26}$

Table 1 shows the mathematical models that describe the normalized ${ }^{177} \mathrm{Lu}-\mathrm{CR}$ experimental emission spectrum; the mathematical models for the two subregions (380 to $600 \mathrm{~nm}$ and 600 to $900 \mathrm{~nm}$ ) are indicated.

\subsection{Determination of the Optical Transmission ${ }^{177} \mathrm{Lu}-\mathrm{CR}$ in Tissue}

In general, light transport by Monte Carlo method is performed in models that consider turbid homogeneous media or multilayer. ${ }^{27,28}$ But if it is considered that the refraction index is similar for different biological tissues (skin, brain, liver, kidneys, etc.), then a homogeneous media can be simulated, using absorption and scattering coefficients that represent various tissues and blood as a "generic tissue."12,27,29 In this work, one layer of such tissue was considered in the simulation.

In Fig. 5, the normalized values of the light transmission for different tissue thicknesses obtained by Monte Carlo simulation are presented. As expected, greater light transmission was obtained in the 600- to $900-\mathrm{nm}$ wavelength interval, which falls within the interval of the tissue optical window (600 to $1100 \mathrm{~nm}$ ), where the tissues become optically transparent. ${ }^{12}$

Considering the general light transmission profile as a function of the wavelength at different tissue thicknesses (Fig. 5), as well as the experimental ${ }^{177} \mathrm{Lu}-\mathrm{CR}$ emission spectrum, the efficiency of the Cerenkov light transmission generated by ${ }^{177} \mathrm{Lu}$ at different tissue thicknesses and wavelengths was obtained (Fig. 6).

Figure 7 shows the percentage of the total emergent photons in the tissue optical window. It was calculated as the sum of the integral of each ${ }^{177} \mathrm{Lu}-\mathrm{CR}$ transmission efficiency function obtained at different tissue thickness in the 600- to 900-nm range. Given the fact that the main organs of mice have an average depth of $1.25 \mathrm{~cm}$ (liver, kidney, spleen, intestine, lungs, heart, and induced tumors) $)^{30}$ it can be seen from Fig. 7 that about $20 \%$ of the photons produced emerged from tissue. This percentage sustains the potential acquisition of images from the CR, which supports the biokinetics of ${ }^{177} \mathrm{Lu}$-radiopharmaceuticals in the main organs of mice. Nevertheless, the imaging correction factors due to the overlapping of organs must be determined for improving the quantification by the optical imaging of the parameters related to the ${ }^{177} \mathrm{Lu}$-radiopharmaceutical biokinetics.

The function fitting of the percentage of total emergent photons from the tissue optical window with respect to the depth is represented in Eq. (15), where $A=33.51, B=41.60$, $C=42.01, D=1.3187, a=0.12, b=0.95$, and $c=0.95$. 

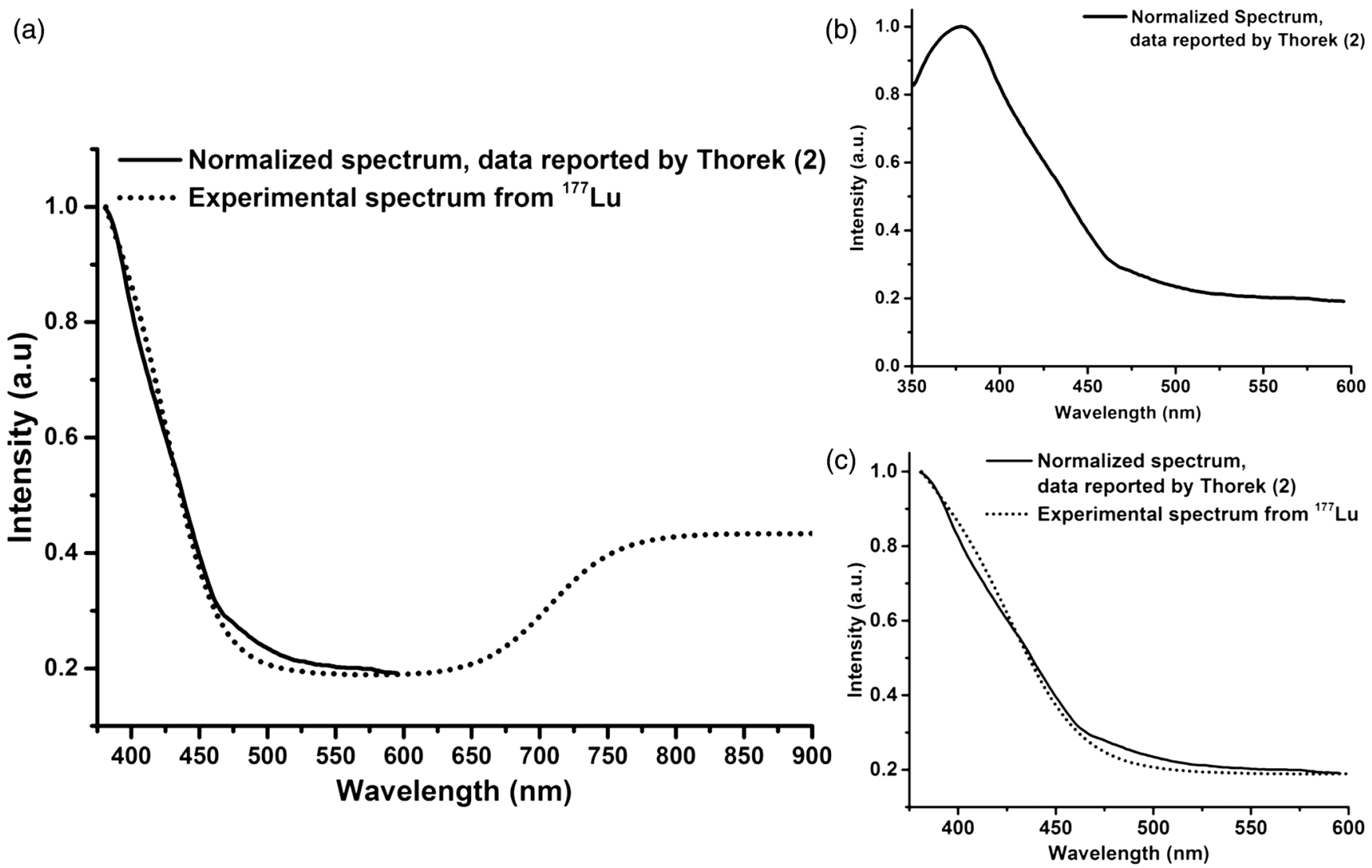

Fig. 4 (a) Normalized emission spectrum of ${ }^{68} \mathrm{Ga}$ reported by Thorek et al. ${ }^{2}$ (b) Normalized emission spectrum of ${ }^{68} \mathrm{Ga}$ obtained from the original spectrum, which was reported by Thorek et al. $^{2}$ (c) Comparison between both CR spectra obtained in the spectral range from 380 to $600 \mathrm{~nm}$.

Table 1 Normalized mathematical model of the experimental ${ }^{177} \mathrm{Lu}-$ CR emission spectrum in the spectral ranges of 350 to $600 \mathrm{~nm}$ and 600 to $900 \mathrm{~nm}$.

\begin{tabular}{lcr}
\hline & Mathematical model $I(\lambda)$ & \\
\hline & $I(\lambda)=A+\frac{B-C}{1+10^{(a-\lambda)(-b)}}$ & \\
\hline Wavelength $(\mathrm{nm})$ & Constant values & R-square \\
\hline 380 to 600 & $A=0.1876$ \\
& $B=1.0828$ \\
& $C=0.1876$ \\
& $a=423.3840$ \\
& $b=0.0216$ & \\
& $A=0.4292$ & \\
& $B=1.0004$ & \\
& $C=0.4292$ & \\
& $a=707.7825$ \\
$b=0.01768$ & \\
\end{tabular}

The inverse of this function can be considered as the tissue attenuation function; knowing the percentage of the total emergent photons at the tissue depth to be studied, the optical image can be corrected by the attenuation factor

\%total emergent photons (depth)

$$
=A e^{(-\mathrm{depth} / a)}+B e^{(-\mathrm{depth} / b)}+C e^{(-\mathrm{depth} / c)}-D .
$$

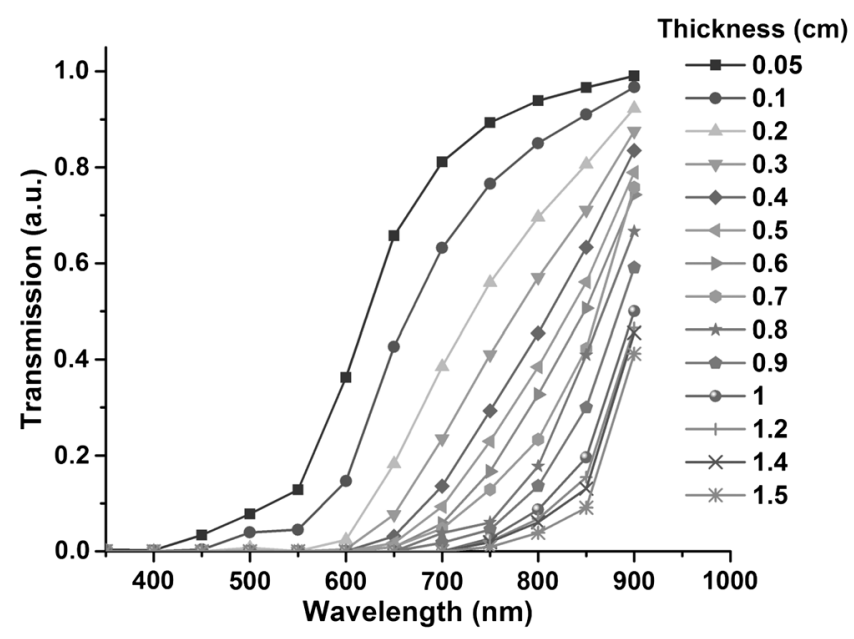

Fig. 5 Light transmission profile for different thicknesses of tissue as a function of the wavelength obtained by Monte Carlo simulation (MCLTmx code).

\section{4 ${ }^{177}$ Lu-CR Image Acquisition and Quantitative Analysis}

The ${ }^{177} \mathrm{Lu}-\mathrm{CR}$ images of mice acquired using the BVX, and reconstructed as per the methodology reported by RamírezNava et al. ${ }^{21}$ at 2 and 24 h, are depicted in Fig. 8; images were also acquired at 4,48 , and $120 \mathrm{~h}$. The quantitative analysis of the ${ }^{177} \mathrm{Lu}-\mathrm{CR}$ images shows that the mean depths of the tumor 


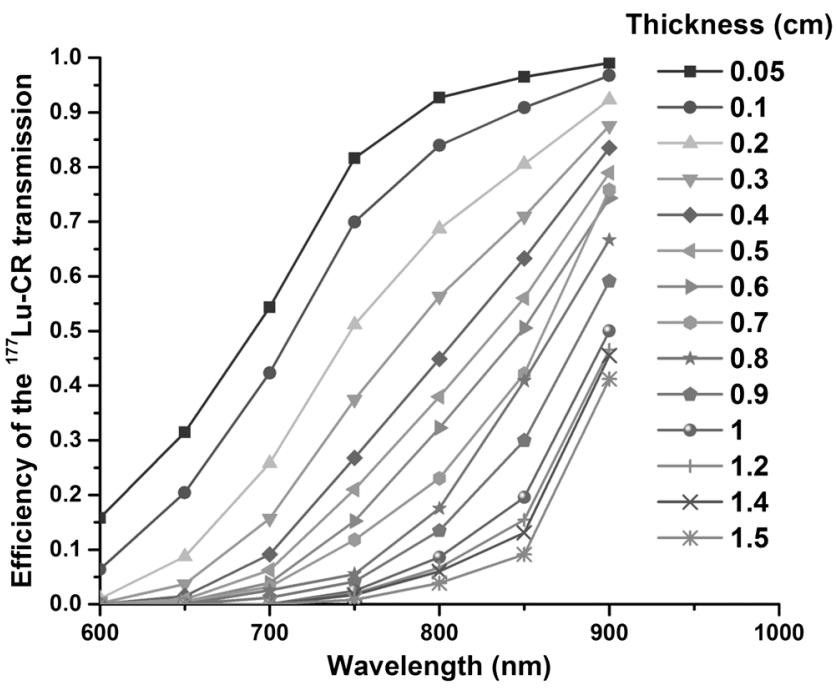

Fig. 6 Cerenkov light transmission profile generated by ${ }^{177} \mathrm{Lu}$ at different tissue thicknesses (efficiency of transmission) obtained by the product of the experimental ${ }^{177} \mathrm{Lu}-\mathrm{CR}$ spectrum [Fig. 4(a)] and the light transmission profile (Fig. 5).

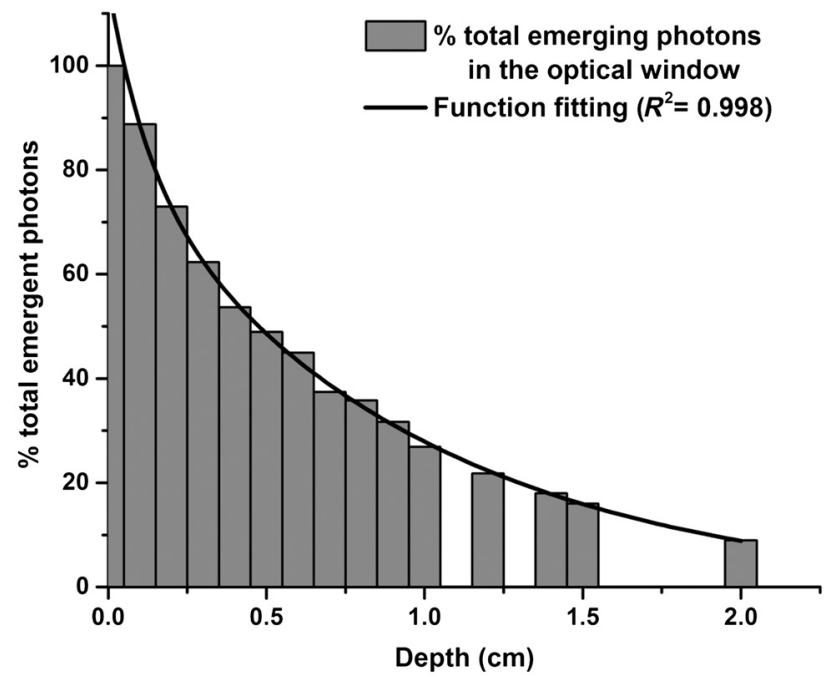

Fig. 7 Percentage of transmission of total photons in the optical spectral window with respect to depth and its function fitting. and kidneys are 0.4 and $0.9 \mathrm{~cm}$, respectively. The number of photons per unit time per unit area $\left(\mathrm{p} / \mathrm{s} / \mathrm{mm}^{2}\right)$ in the kidneys and tumor at different time durations and their correction by the attenuation factor are listed in Table 2.

The total activity in $\mathrm{MBq}$ found in the kidneys and tumor at different time durations (Table 3) was obtained from Eq. (16), which relates the photons per unit time per unit area as a function of the activity. Equation 16 was obtained from the calibration curve of the CCD in-vivo xtreme system as the function of different activities (MBq) (Fig. 9). In Table 3, the biokinetic models in both regions of interest are also presented. It is to be noted that for obtaining high-quality optical images, it is necessary to characterize the CCD, including its limit of detection, dependence of the limit of detection on the production of photons, and its dependence on the initial activity because low signal intensity relative to the CR is a major limitation. Tamura et al. $^{7}$ argue that preclinical instrumentation should focus on maximizing the light generated by a radionuclide source, while minimizing the light loss (by absorbance in the optical path) related to the detector wavelength sensitivity. Solving these shortcomings, the clinical translation to in-vivo models can be accelerated

$\mathrm{p} / \mathrm{s} / \mathrm{mm}^{2}$ (Activity) $=A-B e^{(\text {Activity } / a)}$,

where $A=13321.9803, B=13302.702$, and $a=-18.980$.

Finally, the total number of disintegrations, $N\left(\mathrm{MBq}^{*} \mathrm{~h} /\right.$ $\mathrm{MBq}$ ), in the kidneys and tumor obtained by the integration over time of the biokinetic models are presented in Table 4 . The results obtained in this study using ${ }^{177} \mathrm{Lu}-\mathrm{CR}$ imaging and Monte Carlo simulation do not significantly differ $(<3 \%)$ with those obtained from an ex-vivo model that used the same ${ }^{177} \mathrm{Lu}$ radiopharmaceutical reported by Aranda-Lara et al. ${ }^{20}$

Various biomolecules, peptides, and proteins labelled as ${ }^{18} \mathrm{~F}$, ${ }^{90} \mathrm{Y},{ }^{64} \mathrm{Cu},{ }^{68} \mathrm{Ga},{ }^{89} \mathrm{Zr},{ }^{177} \mathrm{Lu}$, etc., are administered for specific diseases. These radiopharmaceuticals, which have demonstrated significant improvement in the condition and life quality of patients, ${ }^{31,32}$ can also generate CR. Several authors have mentioned that in addition to their use in PET or SPECT, they have significant potential for employment in preclinical studies using optical image evaluation techniques. Owing to the absorption of the CR generated in human tissue, optical imaging by $\mathrm{CR}$ cannot be used in clinical studies. Nevertheless, optimal

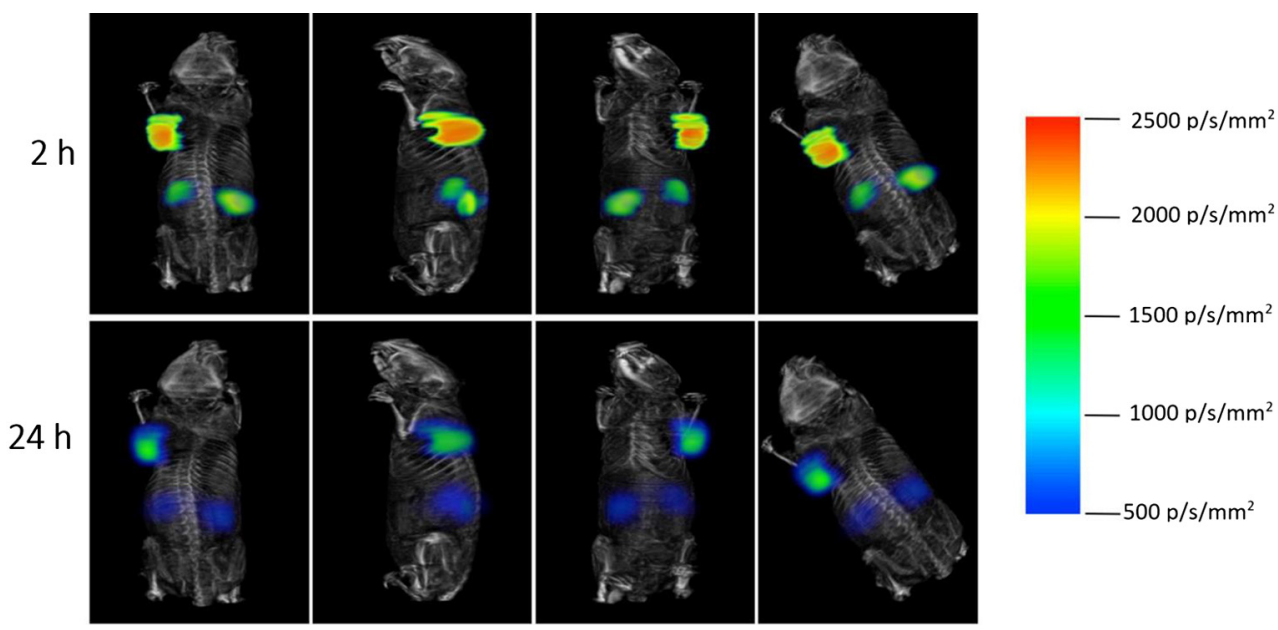

Fig. 8 Tridimensional fusion of ${ }^{177} \mathrm{Lu}-\mathrm{CR}$ (green color) and $\mathrm{x}$-ray images of mice acquired in a Bruker's in-vivo xtreme system at times 2 and $24 \mathrm{~h}$. 
Table 2 Number of photons per unit time per unit area $\left(\mathrm{p} / \mathrm{s} / \mathrm{mm}^{2}\right)$ for tumor and kidneys at different times obtained from the optical images and their correction by the attenuation factor.

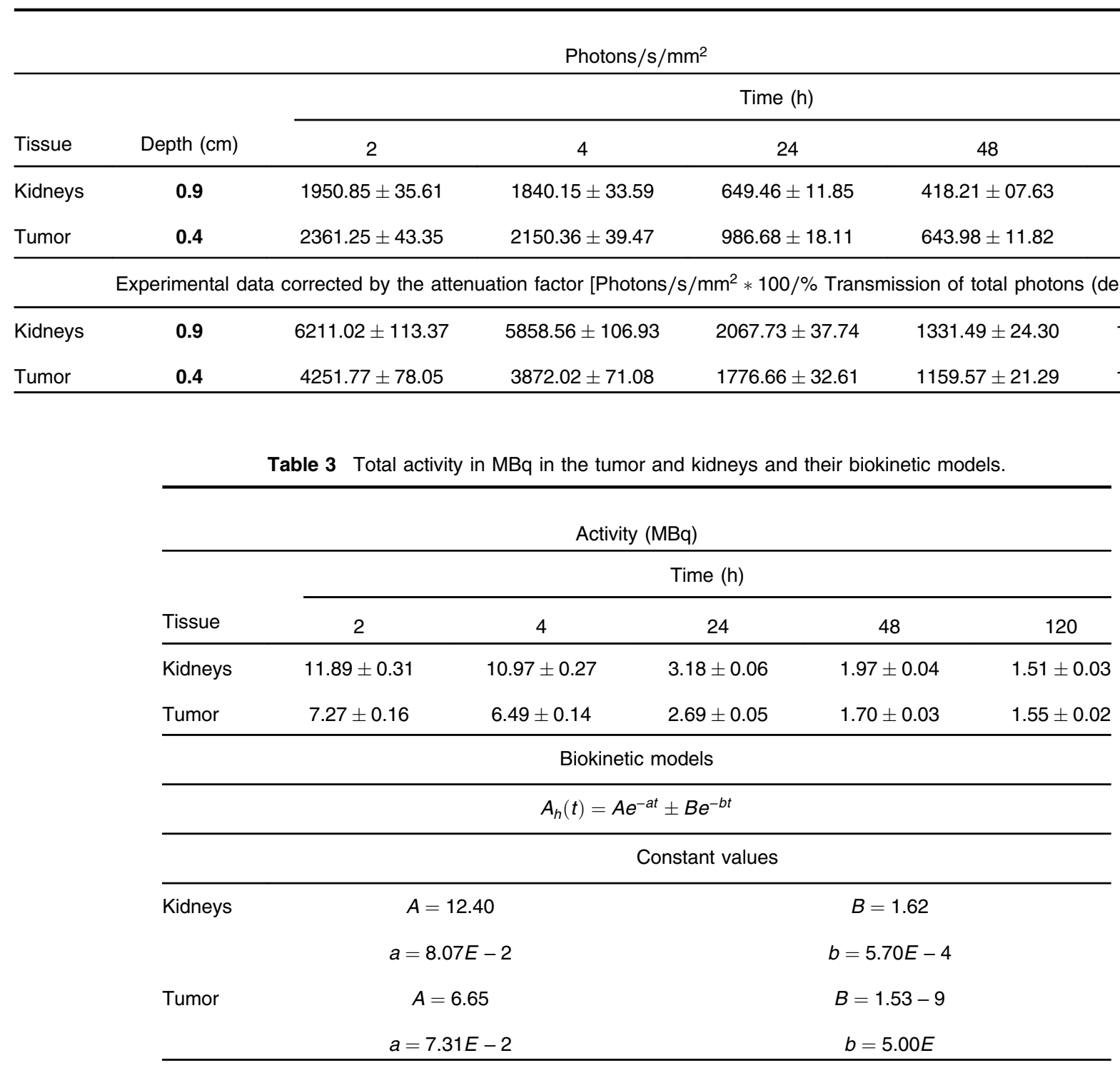

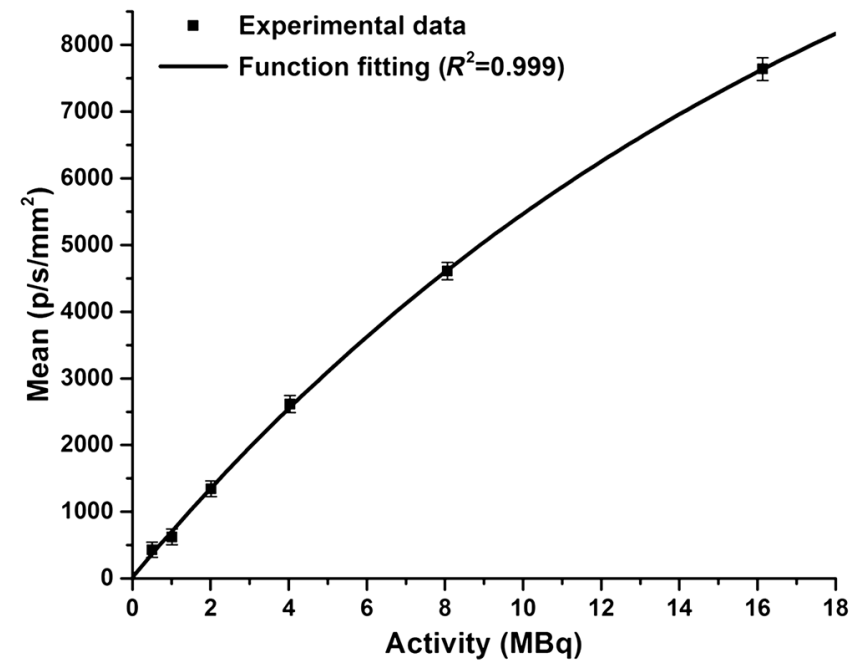

Fig. 9 Calibration of the CCD in-vivo xtreme system and function fitting.
Table 4 Total number of disintegrations $N\left(\mathrm{MBq}^{*} \mathrm{~h} / \mathrm{MBq}\right)$ obtained in this research by optical imaging and the comparison with that reported by Aranda-Lara et al. ${ }^{20}$ from an ex-vivo method.

\begin{tabular}{lcc}
\hline Tissue & $\begin{array}{c}N=\int_{t=0}^{t=0} A_{h}(t) \mathrm{d} t \\
\text { Total disintegrations } \\
\left(\mathrm{MBq}^{*} \mathrm{~h} / \mathrm{MBq}\right)\end{array}$ & $\begin{array}{c}{ }^{*} N\left(\mathrm{MBq}^{*} \mathrm{~h} / \mathrm{MBq}\right) \\
\text { Reported by } \\
\text { Aranda-Lara et al. }{ }^{20}\end{array}$ \\
\hline Kidneys & $4.88 \pm 0.19$ & $4.75 \pm 0.67$ \\
Tumor & $4.40 \pm 0.09$ & $4.33 \pm 0.31$ \\
\hline
\end{tabular}

characterization of the $\mathrm{CR}$ images can improve the image resolution because $\mathrm{CR}$ generation takes place close to the point where radioactive disintegration occurs and therefore, less image reconstruction would be required in comparison with the PET reconstruction techniques. ${ }^{7,8,33}$

It has been reported that the CR optical image technique is better characterized for evaluating tissue under hypoxia. ${ }^{34}$ This 
technique is the only one that can be corroborated by other imaging techniques, such as the PET or SPECT (different imaging principle). Moreover, it has additional advantages compared to the traditional nuclear medicine imaging techniques in terms of the cost, rapidity of image acquisition, and image evaluation. ${ }^{7,8}$

\section{Conclusions}

This study presented a methodology based on the theoretical and experimental characterizations of the generation and range of ${ }^{177}$ Lu-CR. By Monte Carlo simulation, the absorption and/or attenuation parameters in tissue were established and subsequently, the biokinetic model of the ${ }^{177} \mathrm{Lu}$-radiopharmaceutical was successfully obtained.

The results of experimental characterization showed that $45 \%$ of the total intensity of the ${ }^{177} \mathrm{Lu}-\mathrm{CR}$ emission spectrum corresponds to photons with energies within the tissue optical window. The total percentage of emerging photons as a function of the depth of ${ }^{177} \mathrm{Lu}-\mathrm{CR}$, obtained by Monte Carlo simulation and the experimental spectrum of ${ }^{177} \mathrm{Lu}-\mathrm{CR}$, demonstrated that emergent photons existed even at a tissue depth of $1.25 \mathrm{~cm}$ (average depth of main organs of mice). The results obtained from the preclinical ${ }^{177} \mathrm{Lu}-\mathrm{CR}$ images agreed with the theoretical transmission spectrum and demonstrated that the biokinetics of ${ }^{177} \mathrm{Lu}$-radiopharmaceuticals in the main organs of mice can be acquired from the CR optical images. The results indicate that ${ }^{177} \mathrm{Lu}$ can be applied for the acquisition of optical images by $\mathrm{CR}$ generation in tissue and can be implemented in routine preclinical studies. ${ }^{177} \mathrm{Lu}-\mathrm{CR}$ optical images are an alternative for the preclinical evaluation of new ${ }^{177} \mathrm{Lu}$-radiopharmaceuticals.

\section{Disclosures}

The authors have no relevant financial interests in the manuscript and no other potential conflicts of interest to disclose.

\section{Acknowledgments}

This study was supported by the Mexican National Council of Science and Technology (CONACYT) through the CATEDRAS-CONACYT-ININ-337 and CONACYT-SEP-CB2016-286753 projects. It was carried out as part of the activities of the "Laboratorio Nacional de Investigación y Desarrollo de Radiofármacos, CONACyT (LANIDER-CONACYT)" and the Red-Biofotónica, CONACYT. The financial support of the SIEA-UAEMex through the Grant No 4348/2017/CI is also acknowledged. We would like to thank Editage (www.editage .com) for English language editing.

\section{References}

1. K. Tanha, A. M. Pashazadeh, and B. W. Pogue, "Review of biomedical Čerenkov luminescence imaging applications," Biomed. Opt. Express 6, 3053-3065 (2015).

2. D. L. Thorek et al., "Cerenkov imaging - a new modality for molecular imaging," Am. J. Nucl. Med. Mol. Imaging 2, 163-173 (2012).

3. A. E. Spinelli and F. Boschi, "Novel biomedical applications of Cerenkov radiation and radioluminescence imaging," Phys. Med. 31(2), 120-129 (2015)

4. A. E. Spinelli et al., "Cerenkov radiation allows in vivo optical imaging of positron emitting radiotracers," Phys. Med. Biol. 55, 483-495 (2010).

5. X. Ma, J. Wang, and Z. Cheng, "Cerenkov radiation: a multi-functional approach for biological sciences," Front. Phys. 2, 4 (2014).

6. B. J. Beattie et al., "Quantitative modeling of Cerenkov light production efficiency from medical radionuclides," PLoS One 7, e31402 (2012).
7. R. Tamura, E. C. Pratt, and J. Grimm, "Innovations in nuclear imaging instrumentation: Cerenkov imaging," Semin. Nucl. Med. 48(4), 359-366 (2018).

8. R. Robertson et al., "Optical imaging of Cerenkov light generation from positron-emitting radiotracers," Phys. Med. Biol. 54(16), N355-N365 (2009).

9. M. Luna-Gutiérrez et al., "177Lu-labeled monomeric, dimeric and multimeric RGD peptides for the therapy of tumors expressing $\alpha(\nu) \beta(3)$ integrins," J. Label. Compd. Radiopharm. 55, 140-148 (2012).

10. N. Jimenez-Mancilla et al., "Multifunctional targeted radiotherapy system for induced tumours expressing gastrin-releasing peptide receptors," Curr. Nanosci. 8, 193-201 (2012).

11. M. Cremonesi et al., "Correlation of dose with toxicity and tumour response to 90Y- and 177Lu-PRRT provides the basis for optimization through individualized treatment planning," Eur. J. Nucl. Med. Mol. Imaging 45(13), 2426-2441 (2018).

12. S. L. Jacques, "Optical properties of biological tissues: a review," Phys. Med. Biol. 58, R37 (2013).

13. G. S. Mitchell et al., "In vivo Cerenkov luminescence imaging: a new tool for molecular imaging," Philos. Trans. R. Soc. A Math. Phys. Eng. Sci. 369(1955), 4605-4619 (2011).

14. Z. Hu et al., "Experimental Cerenkov luminescence tomography of the mouse model with SPECT imaging validation," Opt. Express 18(24), 24441-24450 (2010).

15. H. Liu et al., "Molecular optical imaging with radioactive probes," PLoS One 5(3), e9470 (2010).

16. H. Cember and T. E. Johnson, Introduction to Health Physics, 4 ed., McGraw-Hill Education, New York (1969).

17. E. Torres-García et al., "A new Monte Carlo code for light transport in biological tissue," Med. Biol. Eng. Comput. 56(4), 649-655 (2018).

18. X. Ma et al., "Bulk optical parameters of porcine skin dermis at eight wavelengths from 325 to 1557 nm," Opt. Lett. 30(4), 412-414 (2004).

19. T. Lister, P. A. Wright, and P. H. Chappell, "Optical properties of human skin,” J. Biomed. Opt. 17(9), 090901 (2012).

20. L. Aranda-Lara et al., "Synthesis and evaluation of Lys1 $(\alpha, \gamma$-Folate) Lys3(177Lu-DOTA)-Bombesin(1-14) as a potential theranostic radiopharmaceutical for breast cancer," Appl. Radiat. Isot. 107, 214-219 (2016).

21. G. J. Ramírez-Nava et al., "Multimodal molecular 3D imaging for the tumoral volumetric distribution assessment of folate-based biosensors," Med. Biol. Eng. Comput. 56(7), 1135-1148 (2018).

22. C. Li, G. S. Mitchell, and S. R. Cherry, "Cerenkov luminescence tomography for small-animal imaging," Opt. Lett. 35(7), 1109-1111 (2010).

23. E. Brayfindley et al., "Automated defect detection in spent nuclear fuel using combined Cerenkov radiation and gamma emission tomography data," Nucl. Technol. 204, 343-353 (2018).

24. "RADAR (RAdiation Dose Assessment Resource)," http://www .doseinfo-radar.com/ (accessed 23 February 2018).

25. E. C. Pratt, T. M. Shaffer, and J. Grimm, "Nanoparticles and radiotracers: advances toward radionanomedicine," Wiley Interdiscip. Rev. Nanomed. Nanobiotechnol. 8(6), 872-890 (2016).

26. A. Ruggiero et al., "Cerenkov luminescence imaging of medical isotopes," J. Nucl. Med. 51(7), 1123-1130 (2010).

27. S. A. Prahl et al., "A Monte Carlo model of light propagation in tissue," Proc. SPIE 10305, 1030509 (1989).

28. Y. Wang, "Accurate Monte Carlo simulation of frequency-domain optical coherence tomography," 35(4), e3177 (2019).

29. L. Wang, S. L. Jacquesa, and L. Zhengb, "MCML-Monte Carlo modeling of light transport in multi-layered tissues," Comput. Methods Programs Biomed. 47, 131-146 (1995).

30. W. H. Miller et al., "Evaluation of beta-absorbed fractions in a mouse model for $90 \mathrm{Y}, 188 \mathrm{Re}, 166 \mathrm{Ho}, 149 \mathrm{Pm}, 64 \mathrm{Cu}$, and $177 \mathrm{Lu}$ radionuclides," Cancer Biother. Radiopharm. 20(4), 436-449 (2005).

31. A. O. J. Garc1, L. D1, and Á. L. Rgd, "Preparation and in vitro evaluation of 177 Lu-iPSMA-RGD as a new heterobivalent radiopharmaceutical," J. Radioanal. Nucl. Chem. 314(3), 2201-2207 (2017).

32. C. Santos-Cuevas et al., "177Lu-DOTA-HYNIC-Lys(Nal)-Urea-Glu: biokinetics, dosimetry, and evaluation in patients with advanced prostate cancer," Contrast Media Mol. Imaging 2018, 1-10 (2018).

33. A. E. Spinelli and F. Boschi, "Optimizing in vivo small animal Cerenkov luminescence imaging,” J. Biomed. Opt. 17(4), 040506 (2012). 
34. E. Desvaux et al., "Cherenkov luminescence imaging is a fast and relevant preclinical tool to assess tumour hypoxia in vivo," EJNMMI Res. 8(1), 111 (2018).

Nallely P. Jiménez-Mancilla received her BSc degree in physics (2007), MSc degree in medical physics (2010), and PhD in health sciences (2014) from the Autonomous University of the State of Mexico. Since 2015, she has worked at the National Council of Science and Technology (Consejo Nacional de Ciencia y Tecnología, CONACYT) and National Institute of Nuclear Research (Instituto Nacional de Investigaciones Nucleares, ININ). She is a researcher in applied physics for therapy and molecular imaging of radiolabeled probes and nanosystems.

Keila Isaac-Olivé received a BSc degree in radiochemistry from the Higher Institute of Nuclear Science and Technology, Havana-Cuba (1998), BSc degree in chemistry from the University of Havana (1999), and MSc (2001) and PhD (2005) from Dalhousie University, Nova Scotia, Canada. She was a postdoctoral fellow from 2007 to 2009 at the Physics Institute, National Autonomous University of Mexico, Mexico. In 2009, she joined the faculty of the Medical Physics Program at the Autonomous University of the State of Mexico (Toluca, Mexico). She is an expert in analytical and radioanalytical techniques, particularly in the use and applications of radionuclides.

Eugenio Torres-García received his BSc degree in physics (2001), and his $\mathrm{PhD}$ in medical physics (2007) from the Autonomous University of the State of Mexico. Since 2007, he has worked in the medical physics program of the Autonomous University of the State of Mexico. $\mathrm{He}$ is a researcher in medical imaging, ionizing radiation dosimetry, nuclear medicine, and Monte Carlo simulation.

Miguel A. Camacho-López received a BSc degree in physics (1993), MSc in physics (1996), and $\mathrm{PhD}$ in physics (Heriot-Watt University, Scotland UK, 2003). He was a postdoctoral fellow at the Liquid Crystal Institute, Kent State University, USA (2000-2002) and a postdoctoral research fellow at the School of Physics and Astronomy, University of St Andrews, Scotland, UK (2002-2004). In 2004, he joined the faculty of the Medical Physics Program at the Autonomous University of the State of Mexico. $\mathrm{He}$ is an expert in biophotonics and the optical properties of nanomaterials.

Gerardo J. Ramírez-Nava received his BSc degree in biomedical engineering (2013) and MSc in medical physics (2016). Since 2017, he has been a PhD student of the nanosciences and micronanotechnologies program at the National Polytechnic Institute of Mexico. His research focuses on image processing, nuclear imaging and dosimetry.

Héctor J. Mendoza-Nava received his BSc degree in physics (2009), MSc in medical physics (2012), and PhD in health sciences (2017) from the Autonomous University of the State of Mexico (UAEM). $\mathrm{He}$ works at the National Institute of Nuclear Research in Mexico (Instituto Nacional de Investigaciones Nucleares, ININ), Metrology Department. $\mathrm{He}$ is a researcher in nuclear medicine and metrology of ionizing radiation in medical applications. 